\title{
Crecimiento del pato criollo suplementado con óxidos naturales y vitamina $\mathbf{E}$
}

\section{Growth of creole duck supplementated with natural oxides and vitamin $\mathbf{E}$}

\author{
Manuel Paredes ${ }^{1 *}(\mathbb{D})$, Asunta León ${ }^{1}$, Wuesley Alvarez ${ }^{1}$ (D) Luis Vilela ${ }^{1}$, Luis Vallejos ${ }^{1}(\mathbb{D})$, José Mantilla ${ }^{1}$ (D)
}

\section{RESUMEN}

El experimento se llevó a cabo en la granja avícola de la Universidad Nacional de Cajamarca, durante siete semanas para determinar los efectos de la suplementación combinada de una mezcla de óxidos naturales (ON) y vitamina $\mathrm{E}$ (VE) sobre el rendimiento en crecimiento y el peso relativo de los órganos inmunes en patos alimentados con dietas que contienen maíz contaminado naturalmente con micotoxinas, principalmente aflatoxinas $\left(\mathrm{AFB}_{1}\right)$. Se evaluaron un total de 400 patos machos asignados al azar a 1 de 4 tratamientos en un arreglo factorial de 2 × 2 con 2 niveles de $\mathrm{ON}(0$ y $100 \mathrm{~g} / \mathrm{kg}$ ) y 2 niveles de VE (0 y $100 \mathrm{mg} / \mathrm{kg})$. Se tuvo 5 repeticiones (corrales) por tratamiento y 20 aves por corral. Los patos alimentados con dietas sin ON tuvieron ganancias de peso más bajas $(\mathrm{p}<0,05)$, y mayores tasas de mortalidad $(p<0,05)$ durante todo el experimento. La suplementación de VE mejoró $(p<0,05)$ conversión alimenticia. Los patos alimentados con dietas sin ON tuvieron pesos relativos mayores $(\mathrm{p}<0,05)$ de hígado, bazo y bolsa de fabricio pero menor $(\mathrm{p}<0,05)$ peso de timo. En conclusión, la alimentación en base a maíz contaminado con $\mathrm{AFB}_{1} \sin \mathrm{ON}$ causó efectos adversos en el rendimiento del crecimiento y el peso relativo de los órganos inmunes en los patos criollos, mientras que la adición dietética de VE contrarrestó parcialmente los efectos negativos de AFB1 en el crecimiento y la tasa de mortalidad.

Palabras clave: óxidos naturales, vitamina E, pato criollo, crecimiento, órganos inmunes.

\begin{abstract}
The experiment was carried out in the poultry farm of the National University of Cajamarca, during seven weeks to determine effects of a combined natural oxides (ON) and vitamin E (VE) supplementation on growth performance and relative organ weight in creole ducks fed diets containing maize naturally contaminated with mycotoxins, primarily aflatoxin $\left(\mathrm{AFB}_{1}\right)$. A total of 400 ducks were randomly assigned to 1 of 4 treatments in a $2 \times 2$ factorial arrangement with 2 levels of ON $(0$ or $100 \mathrm{~g} / \mathrm{kg})$ and 2 VE levels $(0$ or $10 \mathrm{mg} / \mathrm{kg})$. There were 5 replications (pens) per treatment and 20 birds per pen. Ducks fed $\mathrm{AFB}_{1}$ contaminated maize diets and out $\mathrm{ON}$ had lower $(\mathrm{p}<0.05)$ gain body weight and greater $(p<0.05)$ death rates during the whole experiment. The supplementation of VE increased $(p<0.05)$ feed conversion ratio. Ducks fed without oxides in diet had greater $(p<0.05)$ relative weights of liver, spleen and bursa of fabricius but lower $(\mathrm{p}<0.05)$ thymus. In conclusion, feeding $\mathrm{AFB}_{1}$ contaminated maize without oxides supplementation caused adverse effects on growth performance and relative immune organ weight in ducks, while dietary addition of VE partially counteracted the negative effects of $\mathrm{AFB}_{1}$ on growth and death rate.
\end{abstract}

Keywords: natural oxides, vitamin E, creole duck, growth, immune organs

\footnotetext{
${ }^{1}$ Universidad Nacional de Cajamarca, Departamento Académico de Ciencias Pecuarias, Cajamarca, Perú.

*Autor de Correspondencia, e-mail: mparedes@unc.edu.pe
} 


\section{INTRODUCCIÓN}

Las micotoxinas en la alimentación del pato pueden producir lesiones orales, disminución de la ingesta de alimento, afectando incrementos de peso corporal (Chen et al., 2016), además de una deficiente digestibilidad de los nutrientes (Yang et al., 2014). También las micotoxinas afectan la respuesta blastogénica de los linfocitos por el agotamiento de estos en el bazo y la bolsa de fabricio (Rafai et al., 2000).

Existen una serie de micotoxinas, dentro de las cuales las aflatoxinas (AF), tienen toxicidad caracterizada por sus efectos cancerígenos, mutagénicos, teratogénicos y de inhibición del crecimiento (Oğuz et al., 2000), causando importantes cambios microscópicos en el hígado, como hepatomegalia, palidez, degeneración hidrópica, cambio graso, hiperplasia de las vías biliares y fibrosis periportal, además de lesiones de riñón y bazo (Oğuz et al., 2003). La AF del tipo $\mathrm{B}_{1}$, es la micotoxina más potente, que incluso se puede encontrar en el hígado de aves de engorde expuestas a AF luego de ser beneficiadas (Denli et al., 2009); por ser el hígado y el riñón los principales órganos involucrados en la desintoxicación de $\mathrm{AFB}_{1}$ (Hussain et al., 2010).

$\mathrm{Al}$ respecto se ha evaluado la inclusión en el alimento de bentonita de sodio y monensina para disminuir la aflatoxicosis en las aves habiéndose reducido los residuos en hígado de pollos de engorde sacrificados, que consumieron alimento contaminado con niveles de $50 \mu \mathrm{g} / \mathrm{kg}$ de dieta con $\mathrm{AFB}_{1}$ (Magnoli et al., 2011). Los aluminosilicatos de calcio y sodio hidratado también han reducido el efecto residual de $\mathrm{AFB}_{1}$ en hígado y riñones de aves, sin embargo, no han sido muy eficaces en evitar daños histopatológicos hepáticos en pollos de engorde (Neeff et al., 2013). El uso de arcillas como adsorbentes de micotoxinas en la dieta del pato Pekín evita menores tasas de digestibilidad de algunos nutrientes (Yang et al., 2014). La adición de $0,1 \%$ de arcillas adsorbentes puede proteger contra los efectos perjudiciales causados por maíz contaminado con $\mathrm{AFB}_{1}$ en dietas para patos (Wan et al., 2013).

La introducción de una variedad de materiales o mez- clas complejas para producir la unión de AF ha dado lugar a incluir en la dieta de las aves productos denominados enterosorbentes, aglutinantes, secuestrantes, moléculas interceptoras, agentes de captura o adsorbentes de micotoxinas; los cuales pueden contener arcillas de esmectita, zeolitas, caolinita, mica, sílice, carbón y diversos componentes biológicos (Murugesan et al., 2015). Del mismo modo se ha demostrado que la vitamina $\mathrm{E}$ es un buen coadyuvante para evitar efectos colaterales provocados por la aflatoxicosis en patos (He et al., 2013). Además, está demostrado que la suplementación de vitamina E en el pato Pekín mejora las ganancias de peso durante las primeras semanas de vida debido a su capacidad antioxidante e inmunológica (Xie et al., 2018).

El presente trabajo, se orientó a determinar el ritmo de crecimiento del pato criollo (Cairina moschata L) alimentado con una dieta que contiene maíz amarillo molido y contaminado de manera natural con aflatoxinas $\mathrm{B}_{1}$, suplementado con una mezcla de óxidos naturales $(\mathrm{ON})$ y vitamina $\mathrm{E}$ (VE) con la finalidad de atenuar los efectos negativos de la $\mathrm{AFB}_{1}$ sobre los parámetros de crecimiento y peso relativo de los órganos inmunes del pato.

\section{MATERIAL Y MÉTODOS}

Origen del maíz contaminado y análisis de micotoxinas Se obtuvo la suficiente cantidad de maíz para todo el experimento, el que fue trasportado desde la provincia de Trujillo hasta la ciudad de Cajamarca vía terrestre; almacenándose molido y sobre piso de cemento durante dos semanas, para provocar proliferación de hongos y producción de micotoxinas, luego de lo cual se realizó el análisis del maíz para aflatoxinas $B_{1}$ e inmediatamente se lo utilizó en la preparación del pienso, al que se le añadió Fungiban (ácido propiónico) como antimicótico. Se conservó una muestra de maíz molido adicionado con $0.1 \%$ de antimicótico para su posterior análisis luego de 28 días. La técnica de análisis de AF se ciñó a lo indicado por Adrian et al. (2000). 


\section{Manejo de las aves y dietas experimentales}

El experimento se llevó a cabo en la granja avícola de la Facultad de Ingeniería en Ciencias Pecuarias (FICP), de la Universidad Nacional de Cajamarca (UNC). Un total de 400 patos Criollos (Cairina moschata $L$ ) machos de veintiún días de edad con un peso corporal inicial promedio de $747 \mathrm{~g}$ fueron asignados al azar en un arreglo factorial de 2 X 2, formándose los siguientes tratamientos: (1) $\mathrm{S} 1$ : dieta con $0 \mathrm{~g}$ de $\mathrm{ON} / \mathrm{kg}$ y $0 \mathrm{mg}$ de VE/kg; (2) S2: $0 \mathrm{~g}$ de ON/kg y $100 \mathrm{mg}$ de $\mathrm{VE} / \mathrm{kg}$; (3) S3: $100 \mathrm{~g}$ de ON/kg y 0 de mg VE/kg; (4) $\mathrm{S} 4: 100 \mathrm{~g}$ de $\mathrm{ON} / \mathrm{kg}$ y $100 \mathrm{mg}$ de $\mathrm{VE} / \mathrm{kg}$. Se tuvo 5 corrales por tratamiento y 20 patos por corral.

Los ON contienen dióxido de silicio, óxido de aluminio, óxido de hierro, óxido de calcio, óxido de magnesio, óxido de sodio y óxido de potasio. El product es distribuido por Phartec SAC y comercializado como Toxibond como producto adsorbente de micotoxinas. La VE fue suplementada a partir de un aditivo producido por DSM Nutritional Products.

Se utilizó la fórmula alimenticia para pato criollo de 21 días hasta 70 días de edad. La dieta (Tabla 1) fue formulada para cubrir o exceder los requerimientos establecidos por NRC (1994) para patos en crecimiento. Se tuvo cuatro dietas a las que se le adicionó ON o VE según lo establecido en cada tratamiento, por lo que se tuvo dietas S1, S2, S3 Y S4 las que variaron solamente por la inclusión de ON y VE. Las dietas fueron suministradas en forma de harina y se proporcionó pienso y agua ad libitum a lo largo del experimento.

\section{Medición del crecimiento del pato y pesaje de órga- nos inmunes}

Se pesaron los patos y se registró el consumo de alimento en los días 21 y 70 de edad, inicio y final del experimento, se calcularon ganancia media diaria (GMD), ingesta diaria de alimento (IDA) y conversion alimenticia (CA). Los patos muertos se pesaron y la cantidad de muertes fue registrada. La mortalidad se expresó como un porcentaje respecto de las aves iniciales por cada tratamiento.

Al final del experimento, se seleccionaron al azar 2
Tabla 1. Composición de la dieta para pato criollo de 4 a 10 semanas de edad (en base fresca)

\begin{tabular}{cc}
\hline Ingredientes (\%) & \\
\hline Maíz amarillo contaminado $^{1}$ & 62,5 \\
Afrecho de trigo & 3,5 \\
Torta de soya 44\% & 27,0 \\
Aceite de palma & 3,5 \\
Carbonato de calcio & 1,1 \\
Fosfato dicálcico & 1,6 \\
Sal común & 0,4 \\
DL Metionina & 0,1 \\
Cloruro de colina $60 \%$ & 0,1 \\
Premix vitaminas y microminerales ${ }^{2}$ & 0,1 \\
Antimicótico ${ }^{3}$ & 0,1 \\
\hline TOTAL & 100,0 \\
\hline Contenido nutricional calculado & \\
\hline Materia seca, \% & 88,1 \\
Proteína cruda, \% & 18,0 \\
EM, kcal/kg & 3052 \\
Lisina, \% & 0,87 \\
P, \% & 0,60 \\
Ca, \% & 0,90 \\
\hline
\end{tabular}

1 Maíz contaminado naturalmente con Aflatoxina B1 a razón de $73,14 \mu \mathrm{g} / \mathrm{kg}$ (en el día 0 ) y $71,82 \mu \mathrm{g} / \mathrm{kg}$ (en el día 28 )

2 Cada kg de Premix de vitaminas y minerales contiene: Vitamina A 9000000 UI; Vitamina D3 2000000 UI; Vitamina E 8000 UI; Vitamina K3 2 g; Tiamina 1,5 g; Riboflavina 5 g; Piridoxina 1,5 g; Cianocobalamina $9 \mathrm{mg}$; Ácido pantoténico $5 \mathrm{~g}$; Ácido fólico 200 $\mathrm{mg}$; Niacina $25 \mathrm{~g}$. Manganeso $60 \mathrm{~g}$; Zinc $30 \mathrm{~g}$; Hierro $30 \mathrm{~g}$; Cobre $1,5 \mathrm{~g}$; Iodo $1 \mathrm{~g}$; Selenio $100 \mathrm{mg}$; Cobalto $100 \mathrm{mg}$.

3 Nombre comercial: Fungibán, contiene $99 \%$ de ácido propiónico

patos de cada corral y se pesaron individualmente y fueron sacrificados por dislocación cervical. El hígado, bazo, bolsa de fabricius y timo fueron removidos y pesados. El peso del órgano se expresó como un porcentaje del peso corporal.

\section{Análisis estadístico}

Todos los datos, excepto la mortalidad, fueron analizados por ANOVA (SAS Inst. Inc., Cary, NC, USA) Utilizando un arreglo factorial de 2 x 2, con 4 tratamientos, siendo un corral la unidad experimental. El modelo utilizado incluyó los efectos del ON y VE. Se compararon las medias de cada tratamiento utilizando la prueba de Tukey. La variabilidad en los datos se expresa como el error estándar de la media (SEM) y un nivel de probabilidad de $\mathrm{p}<0,05$ fue considerado estadísticamente significativo. 


\section{RESULTADOS Y DISCUSIÓN}

\section{Rendimiento productivo}

Durante las semanas 4 a 10, los patos alimentados con dietas sin ON obtuvieron los pesos finales y GMD más bajos y peores $\mathrm{CA}(\mathrm{p}<0,05)$ y mayor tasa de mortalidad (Tabla 2). La suplementación de VE redujo CA $(\mathrm{p}<0,05)$ y también la tasa de mortalidad en patos alimentados con dietas contaminadas con y sin ON. No se observó efecto interactivo $(\mathrm{p}>0,05)$ en ninguno de los parámetros de crecimiento. Para todo el experimento de 49 días, no se encontraron diferencias en la ingesta de pienso con maíz contaminado $(\mathrm{p}>0,05)$.

Tabla 2. Parámetros de crecimiento del pato criollo macho con suplementación $(\mathrm{S})^{1}$ de óxidos naturales $(\mathrm{ON})$ y vitamina $\mathrm{E}(\mathrm{VE})$ en dietas contaminadas con aflatoxinas $\mathrm{B}_{1}$

\begin{tabular}{|c|c|c|c|c|c|c|c|c|}
\hline & \multirow{2}{*}{ S1 } & \multirow{2}{*}{ S2 } & \multirow{2}{*}{ S3 } & \multirow{2}{*}{ S4 } & \multirow{2}{*}{ SEM } & \multicolumn{3}{|c|}{ p-value } \\
\hline & & & & & & ON & VE & ON x VE \\
\hline Peso inicial, $\mathrm{g}$ & 748,1 & 749,2 & 748,4 & 742,6 & 1,51 & 0,671 & 0,817 & 0,241 \\
\hline Peso final, $\mathbf{g}$ & $3620,5^{\mathrm{b}}$ & $3938,5^{\mathrm{ab}}$ & $4114,9^{a}$ & $4208,7^{\mathrm{a}}$ & 129,46 & 0,021 & 0,045 & 0,190 \\
\hline GMD, g/d & $58,6^{\mathrm{b}}$ & $65,1^{\mathrm{ab}}$ & $68,7^{\mathrm{a}}$ & $70,6^{a}$ & 2,64 & 0,041 & 0,050 & 0,219 \\
\hline IDA, $\mathrm{g} / \mathrm{d}$ & 220,3 & 221,1 & 220,3 & 220,4 & 0,19 & 0,381 & 0,417 & 0,302 \\
\hline CA & $3,76^{\mathrm{a}}$ & $3,4^{b}$ & $3,21^{\mathrm{c}}$ & $3,12^{\mathrm{c}}$ & 0,14 & 0,018 & 0,032 & 0,081 \\
\hline Mortalidad, $\%{ }^{2}$ & 16,0 & 5.0 & 1,0 & 0 & -- & -- & -- & -- \\
\hline
\end{tabular}

SEM: Error estándar de la media. GMD: ganancia media diaria. IDA: ingesta diaria de alimento. CA: conversión alimenticia

${ }_{a, b, c}$ Medias seguidas por letras diferentes en la fila son diferentes según prueba de Tukey $(\mathrm{p}<0,05)$

${ }^{1}$ Combinaciones de suplementación. S1: $0 \mathrm{~g} \mathrm{ON} / \mathrm{kg}$ y $0 \mathrm{mg} \mathrm{VE} / \mathrm{kg} ; \mathrm{S} 2: 0 \mathrm{~g} \mathrm{ON} / \mathrm{kg}$ y $100 \mathrm{mg} \mathrm{VE} / \mathrm{kg} ; \mathrm{S} 3: 100 \mathrm{~g} \mathrm{ON} / \mathrm{kg}$ y 0 mg VE/kg; S4: $100 \mathrm{~g}$ $\mathrm{ON} / \mathrm{kg}$ y $100 \mathrm{mg} \mathrm{VE} / \mathrm{kg}$.

${ }^{2}$ Se estimó como dato único a partir de los 100 patos que conformaron cada tratamiento.

La alimentación con dietas de maíz contaminado con $\mathrm{AFB}_{1}$ deprimió el crecimiento cuando no contenía ON, tal como lo reporta también Chen et al. (2014) quienes determinaron que, a mayores concentraciones de micotoxinas en el alimento, el pato consumía menos alimento. Por otro lado, se observó que la suplementación con VE potencia el crecimiento del pato criollo, reflejado en la superioridad de GMD del ave suplementado con vitamina $\mathrm{E}$ corroborado por $\mathrm{He}$ et al. (2013) quienes encontraron mejores GMD en patos que consumieron dietas que contenían maíz contaminado con $196,8 \mu / \mathrm{kg}$ de $\mathrm{AFB}_{1}$.

En cuanto a consumo de alimento, se debe remarcar que existen investigaciones que han determinado que la presencia en el pienso de aflatoxinas y de las diferentes micotoxinas deprime la ingesta del mismo, observándose tal diferencia cuando los patos consumen dietas con concentraciones crecientes de micotoxinas (Feng et al., 2010; Han et al., 2008). Sin embargo, en nuestro experimento el nivel de contaminación por $\mathrm{AFB}_{1}$ en las cuatro dietas fue el mismo por lo que no se encontraron diferencias en IDA $(\mathrm{p}>0,05)$.

Los ON produjeron en el pato criollo mejor eficiencia en la conversión de alimento corroborándose la fun- ción ligante de micotoxinas de la mezcla de óxidos naturales evitando la absorción de $\mathrm{AFB}_{1}$ hacia el hígado (Murugesan et al., 2015) lo que provocaría alteración de la función hepática e inhibición de la síntesis de proteínas y lipogénesis (Ortatatli y Oguz., 2001). Por su parte la VE también tuvo influencia sobre la CA del pato criollo, mejorándola cuando se suplementó la dieta con $100 \mathrm{~g} / \mathrm{kg}$; esto debido a que la vitamina $\mathrm{E}$ tiene propiedades antioxidantes y es moduladora de la función inmune (Konjufca et al., 2004).

La tasa de mortalidad sin la inclusión de ON y VE en la dieta fue elevada reduciéndose cuando se suplementó con VE, siendo mejor aun cuando a la dieta se le adicionó el adsorbente de micotoxinas $\mathrm{ON}$, y evitándose la mortalidad cuando se suplementó con ambos microingredientes (ON y VE). Se debe resaltar que, si bien la vitamina $\mathrm{E}$ es un buen coadyuvante en la reducción de muertes en el pato criollo bajo condiciones de aflatoxicosis, el ON disminuyó la mortalidad con más notoriedad, encontrándose que la adición de VE redujo la tasa de mortalidad a 5\%, mientras que el $\mathrm{ON}$ disminuyó las bajas hasta $1 \%$. También debe tenerse en cuenta que el maíz estuvo contaminado muy ligeramente por encima del máximo permisible de $\mathrm{AFB}_{1}$, que es de $50 \mu / \mathrm{kg}$ 
(FDA, 2005). Cuando se utiliza maíz en la dieta con una concentración de $\mathrm{AFB}_{1}$ por triplicado respecto del nivel de contaminación del maíz usado en nuestro experimento, se ha encontrado un efecto diluido de la adición dietética de VE sobre la mortalidad (He et al., 2013)

Peso relativo de los órganos inmunes

Dietas de maíz contaminado sin suplementación con
ON aumentó $(\mathrm{p}<0,05)$ los pesos relativos del hígado, bazo y la bolsa de fabricio pero disminuyó $(\mathrm{p}<0,05)$ el del timo (Tabla 3). VE dietética aumentó $(\mathrm{p}<0,05)$ el peso relativo de la bolsa de fabricio. No se observó efecto interactivo entre factores evaluados $(p>0,05)$ en ninguno de los pesos relativos de los órganos inmunes.

Tabla 3. Peso relativo de los órganos inmunes $(\%)^{1}$ del pato criollo macho con suplementación $(\mathrm{S})^{2}$ de óxidos naturales $(\mathrm{ON})$ y vitamina $\mathrm{E}(\mathrm{VE})$ en dietas contaminadas con aflatoxinas B

\begin{tabular}{|c|c|c|c|c|c|c|c|c|}
\hline & \multirow{2}{*}{ S1 } & \multirow{2}{*}{ S2 } & \multirow{2}{*}{ S3 } & \multirow{2}{*}{ S4 } & \multirow{2}{*}{ SEM } & \multicolumn{3}{|c|}{ p-valor } \\
\hline & & & & & & $\mathbf{O N}$ & VE & $\mathbf{O N} \times \mathrm{VE}$ \\
\hline Hígado & $8,48 \mathrm{a}$ & $7,17^{\mathrm{b}}$ & $6,99^{b}$ & $6,04^{\mathrm{c}}$ & 0,50 & 0,038 & 0,042 & 0,231 \\
\hline Bazo & $0,38^{\mathrm{a}}$ & $0,38^{\mathrm{a}}$ & $0,23^{b}$ & $0,23^{b}$ & 0,04 & 0,014 & 0,871 & 0,094 \\
\hline Bolsa de Fabricio & $0,22^{b c}$ & $0,28^{\mathrm{a}}$ & $0,20^{\mathrm{c}}$ & $0,29^{\mathrm{a}}$ & 0,02 & 0,315 & 0,049 & 0,062 \\
\hline Timo & $0,42^{\mathrm{c}}$ & $0,44^{\mathrm{c}}$ & $0,59^{\mathrm{a}}$ & $0,61^{\mathrm{a}}$ & 0,05 & 0,001 & 0,129 & 0,078 \\
\hline
\end{tabular}

SEM: Error estándar de la media

${ }_{a, b, c}$ Medias seguidas por letras diferentes en la fila son diferentes según prueba de Tukey $(\mathrm{p}<0,05)$

${ }^{1}$ Peso de los órganos en relación al peso corporal del pato a las 10 semanas de edad $(n=10)$ según tratamiento

${ }^{2}$ Combinaciones de suplementación. S1: $0 \mathrm{~g} \mathrm{ON} / \mathrm{kg}$ y $0 \mathrm{mg} \mathrm{VE} / \mathrm{kg}$; S2: 0 g ON/kg y $100 \mathrm{mg} \mathrm{VE} / \mathrm{kg} ; \mathrm{S} 3: 100$ g ON/kg y 0 mg VE/kg; S4: 100 g $\mathrm{ON} / \mathrm{kg}$ y $100 \mathrm{mg} \mathrm{VE} / \mathrm{kg}$.

El peso de los órganos inmunes refleja el estado inmunitario del ave (Heckert et al., 2002). Estos órganos son responsables de la producción de inmunoglobulinas (Murugesan et al., 2015). En el presente estudio, el peso relativo del timo fue reducido por la presencia de $\mathrm{AFB}_{1}$ absorbidas cuando no se usó $\mathrm{ON}$ en la dieta, lo que indica que la ausencia de ON deprimió el desarrollo del timo en los patos criollos, sin embargo, cuando se suplementó con VE el tamaño de este órgano mejoró. Por otro lado, la no inclusión de $\mathrm{ON}$ aumentó los pesos relativos del hígado, bazo y bolsa de fabricio, lo que coincide con lo manifestado por Perusia y Rodríguez (2001) acerca del aumento de tamaño de los órganos inmunes ante un caso de micotoxicosis.

\section{CONCLUSIONES}

Dietas a base de maíz contaminado con aflatoxinas en niveles del presente estudio y sin la presencia de óxidos naturales deprimen el crecimiento y la inmunidad del pato criollo macho, al mismo tiempo que aumentan la tasa de mortalidad.

La vitamina $\mathrm{E}$ puede contrarrestar parcialmente los efectos adversos de la dieta de patos criollos contaminado con aflatoxinas, pero sin cumplir cabalmente la función de adsorbente de micotoxinas.

\section{REFERENCIAS BIBLIOGRÁFICAS}

Adrian, J., J. Potus, A. Poiffait, P. Dauvillier. 2000. Análisis nutricional de los alimentos. Zaragoza(España): Editorial Acribia.

Chen X., R. Murdoch, Q. Zhang, D. J. Shafer, y T. J. Applegate. 2016. "Effects of dietary protein concentration on performance and nutrient digestibility in Pekin ducks during aflatoxicosis." Journal Poultry Science 95:834-841 DOI: $10.3382 / p s / p e v 378$

Chen X., N. Horn, P. F. Cotter, y T. J. Applegate. 2014. "Growth, serum biochemistry, complement activity, and liver gene expression responses of Pekin ducklings to graded levels of cultured aflatoxin B1." Journal Poultry Science 93:2028-2036. DOI: 10. 3382/ps. 201403904.

Denli M., J. C. Blandon, M. E. Guynot, S. Salado, y J. F. Perez. 2009. "Effects of dietary AflaDetox on performance, serum biochemistry, histopathological changes, and aflatoxin residues in broilers exposed to aflatoxin B1.” Journal Poultry Science 88:1444-1451.

FDA (Food and Drug Administration). 2005. Feed Contaminants Program. Compliance Pro- 
gram Guidance Manual Program 7371. 003. Washington, DC (USA). U. S. Food and Drug Administration

Feng J., A. Wang y S. Y. Huo. 2011. "Effect of selenium supplementation on growth performance, immune function and endocrine of growing laying ducks". Chin. J. Anim. Nutr 23(10): 1697-1702.

Han X. Y., Q. C. Huang, W. F. Li, J. F. Jiang, y Z. R. Xu. 2008. "Changes in growth performance, digestive enzyme activities and nutrient digestibility of cherry valley ducks in response to aflatoxin B1 level." Journal Livest. Sci. 119 (1-3): 216-220 DOI: 10. 1016/j. livsci. 2008.04.006

He J., KY. Zhang, D. W. Chen, X. M. Ding, G. D. Feng, y X. Ao. 2013. "Effects of vitamin E and selenium yeast on growth performance and immune function in ducks fed maize naturally contaminated with aflatoxin B1." Journal Livestock Science 152: 200-207. DOI: 10. 1016/j. livsci. 2012. 12.018

Heckert R. A., I. Estevez, E. Russek, y R. Pettit. 2002. "Effects of density and perch availability on the immune status of broilers." Journal Poultry Science 81:451-457.

Hussain Z., M. Z. Khan, A. Khan, I. Javed, M. K. Saleemi, S. Mahmood, y M. R. Asi. 2010. "Residues of aflatoxin B1 in broiler meat: Effect of age and dietary aflatoxin B1 levels." Journal Food Chem Toxicol 48 (12):3304-3307. DOI: 10. 1016/j. fct. 2010. 08.016

Konjufca V. K., W. G. Bottje, T. K. Bersi y G. F. Erf. 2004. "Influence of dietary vitamin E on phagocytic functions of macrophages in broilers." Journal Poultry Science 83 (9): 1530-1534 DOI: $10.1093 / \mathrm{ps} / 83.9 .1530$

Magnoli A. P., M. P. Monge, R. D. Miazzo, L. R. Cavaglieri, C. E. Magnoli, C. I. Merkis, A. L. Cristofolini, A. M. Dalcero, y S. M. Chiacchiera. 2011. "Effect of low levels of aflatoxin B1 on performance, biochemical parameters, and aflatoxin B1 in broiler liver tissues in the presence of monensin and sodium bentonite". Journal Poultry Science 90:48-58. DOI: 10.3382/ps. 2010-00971.

Murugesan G. R., D. R. Ledoux, K. Naehrer, F. Berthiller, T. J. Applegate, B. Grenier, T. D. Phillips, y G. Schatzmayr. 2015. "Prevalence and effects of mycotoxins on poultry health and performance, and recent development in mycotoxin counteracting strategies". Journal Poultry Science 94:1298-1315. DOI: 10. $3382 / \mathrm{ps} / \mathrm{pev} 075$

National Research Council. 1994. Nutrient Requirements of Poultry. Washington, DC (USA): National Academy Press

Neeff D. V., D. R. Ledoux, G. E. Rottinghaus, A. J. Bermudez, A. Dakovic, R. A. Murarolli, y C. A. F. Oliveira. 2013. "In vitro and in vivo efficacy of a hydrated sodium calcium aluminosilicate to bind and reduce aflatoxin residues in tissues of broiler chicks fed aflatoxin B1". Journal Poultry Science 92:131-137. DOI: 10.3382/ps. 2012-02510.

Oğuz H., H. H. Hadimli, V. Kurtoglu, y O. Erganis. 2003. "Evaluation of humoral immunity of broilers during chronic aflatoxin (50 and 100 ppb) and clinoptilolite exposure". Rev. Med. Vet. 154:483-486.

Oğuz H., V. Kurtoglu, y B. Coskun. 2000. "Preventive efficacy of clinoptilolite in broiler during chronic aflatoxin (50 and $100 \mathrm{ppb}$ ) exposure". Journal Res. Vet. Sci. 69:197-201.

Ortatatli M. y H. Oguz. 2001. "Ameliorative effects of dietary clinoptilolite on pathological changes in broiler chickens during aflatoxicosis". Journal Res. Vet. Sci. 71: 59-66

Perusia, O. y R. Rodríguez. 2001. "Micotoxicosis". Rev Inv Vet Perú 12 (2): 87-116

Rafai P., H. Pettersson, A. Bata, Z. Papp, R. Gla, S. Tuboly, A. Ványi, y P. Soós. 2000. "Effect of Dietary T-2 Fusariotoxin Concentrations on 
the Health and Production of White Pekin

Duck Broilers". Journal Poultry Science

79:1548-1556.

Wan X. L., Z. B. Yang, W. R. Yang, S. Z. Jiang, G. G. Zhang, S. L. Johnston, y F. Chi. 2013. "Toxicity of increasing aflatoxin B1 concentrations from contaminated corn with or without clay adsorbent supplementation in ducklings". Journal Poultry Science 92:1244-1253. DOI: 10.3382/ps. 2012-02748.

Yang Z. B., X. L. Wan, W. R. Yang, S. Z. Jiang, G. G. Zhang, S. L. Johnston, y F. Chi. 2014. "Effects of naturally mycotoxin-contaminated corn on nutrient and energy utilization of ducks fed diets with or without Calibrin-A". Journal Poultry Science 93:2199-2209. DOI: 10. 3382/ps. 2013-03392.

Xie M., S. Wang, W. Huang, y S. S. Hou. 2018. "Effects of vitamin E on growth performance, tissue $\alpha$-tocopherol, and lipid peroxidation of starter White Pekin ducks". Journal Poultry Science. 97:2139-2143 DOI: 10 . $3382 / \mathrm{ps} / \mathrm{pex} 443$ 